\title{
GAS BUBBLES ELECTROLYTICALLY GENERATED AT MICROCAVITY ELECTRODES (MCE) USED FOR THE MEASUREMENT OF THE DYNAMIC SURFACE TENSION IN LIQUIDS
}

\author{
A. Volanschi, W. Olthuis, P. Bergveld \\ MESA Research Institute, University of Twente, P.O.Box 217, 7500AE Enschede, The Netherlands \\ phone:+31-53-892722, fax:+31-53-328439, email: A.Volanschi@el.utwente.nl
}

\begin{abstract}
SUMMARY
A new method is proposed for the measurement of dynamic surface tension in aqueous solutions. The advantage of this method with respect to the classical method based on sparging is that the use of gas pumps is avoided, resulting in a miniaturised system. This method is based on the in-situ generation of gas bubbles by means of electrolysis at MicroCavity Electrodes (MCE). As a consequence of electrode surface shaping, a single nucleation site for gas bubbles is created. The MCE is used simultaneously as a bubble actuator (generator) and as a bubble size and/or frequency sensor. Measurement results prove the suitability of the electrolytical method for the monitoring of the dynamic surface tension in aqueous solutions.
\end{abstract}

\section{INTRODUCTION}

The measurement of the surface tension in liquids is important for various applications like, for instance, those employing surface active materials for wetting and washing. The surface tension $\sigma$ is defined as half of the free energy due to cohesion of the material in vacuum per unit area $\Delta G[1]$.

$$
\sigma=-\frac{1}{2} \Delta G
$$

Distinction should be made between the static surface tension which characterises the equilibrium situations and the dynamic surface tension, which characterises situations far from equilibrium. Wetting and cleaning processes are usually far from equilibrium and thus the dynamic surface tension of a liquid is the parameter that should be monitored in this situation.

To measure the dynamic surface tension, a surface or interface with changing area is needed. At this surface a dynamic process of coverage with surface active molecules takes place. A possibility to create a changing surface is to grow gas bubbles in the liquid giving rise to a controlled dynamic surface. A reproducible way to generate gas bubbles with a well defined size in a liquid solution is blowing gas through a tube directly into the bulk of the liquid. The method is called sparging and the theory is quite well understood [2]. This method is widely used for dynamic surface tension measurements $[3,4]$. The disadvantage is that a pump with a constant gas flow is needed resulting in quite a big set-up. Concerning the behaviour of gas bubbles generated by sparging, it was observed in literature [5] that the frequency of these bubbles generated at a constant gas flow increases when the surfactant concentration increases (the dynamic surface tension decreases). On the other hand, it was observed that, if the surfactant concentration is maintained constant (constant dynamic surface tension), the bubble frequency increases when the gas flow is increased [6].

An alternative method for gas bubble generation is electrolysis at a metal electrode. The challenge is to generate in-situ monosized bubbles, in a controlled way and only on one specified site, such that the method becomes analogue to sparging. A similar behaviour of the gas bubbles with respect to the electrolysis current and the dynamic surface tension of the solution is expected. A miniaturised system that avoids the use of big and expensive gas pumps should become possible in this way.

The nature of the bubble nucleation processes during electrolysis has to be understood before an artificial nucleation site can be made. In order for the nucleation of gas bubbles to take place, the liquid has to be supersaturated with dissolved gas. The actual value of the supersaturation needed for nucleation is strongly dependent on other parameters like the electrode surface, electrode material, surface tension of the liquid, etc.

Nucleation of gas bubbles in liquids can occur through different mechanisms. One possibility is the homogeneous nucleation where bubbles appear in the bulk of the mother phase in places where the influence of any interface in the system is negligible. The driving force during the homogeneous nucleation is the excess of chemical potential of the liquid phase as compared with that of the gas. Return to a stable situation results in formation of a bubble. The conditions for homogeneous nucleation are fairly infrequently met, reason why the homogeneous nucleation is not as practically significant as the heterogeneous nucleation.

Through heterogeneous nucleation, gas bubbles are formed at a surface of an electrode or at a wall of the containing vessel. The driving force is still an excess of the chemical potential, but the presence of the wall plays an important role through the contact angle. The contact angle of a solid and a liquid phase is defined as the angle of the liquid-gas interface with the solid-liquid interface at the 
contact point. For a non zero contact angle, heterogeneous nucleation is energetically preferred to homogeneous nucleation. In the limiting case when the contact angle is zero, the heterogeneous nucleation ceases to have any energetic advantage on homogeneous nucleation. In the particular case of electrolysis the gas supersaturation is always higher at the electrode-liquid interface than in the bulk liquid because the gas is generated at the electrode surface. Due to this nonuniform concentration profile and to the fact that bubbles nucleate in regions where the supersaturation is high, bubbles will always nucleate at the electrodes and not in the bulk of the liquid. The state of the surface will influence the size and the behaviour of the bubble.

There is still another possibility to get growing bubbles in a liquid, namely when a bubble source exists already in the form of "traces of gas". These traces of gas, called Harvey nuclei [2], may be present in pockets (small cavities) on the surface of an electrode or the wall of the containing vessel and are in equilibrium with the environment. If the concentration of dissolved gas exceeds a maximum value depending on the dimensions of the pockets, the corresponding Harvey nucleus becomes active and a bubble will grow from that pocket.

On any existing flat electrode, the place where the bubbles nucleate is unknown, independent of the active nucleation mechanism. Miniaturisation of the electrode down to the same order of size as bubble size seems to be one solution. The problem is that, even on such microelectrodes, bubbles might form at random places in an uncontrolled manner. For instance, two bubbles can nucleate at the same time and remain so small that they do not coalesce [7]. Detection of the bubbles can be performed by monitoring the fluctuations of either the electrode overpotential or the electrode impedance [8].

In this paper, an alternative method is proposed for the measurement of the dynamic surface tension in aqueous solutions that uses gas bubbles generated in-situ by electrolysis. Electrode surface shaping is proposed to generate bubbles on a specified site and with a reproducible size by means of electrolysis. The surface shaping is used to generate the needed supersaturation gradients of dissolved gas that lead to the heterogeneous nucleation of a gas bubble on one specified place. MicroCavity Electrodes (MCEs) are proposed for this purpose. The detection of the bubbles was performed with the same electrodes, by means of overpotential or impedance measurements. The frequency of the bubbles was used for the monitoring of the dynamic surface tension of an aqueous solution and the results are presented in this paper.

\section{EXPERIMENTAL}

\section{MicroCavity Electrodes (MCE)}

The shape of the cavity of the MCEs was chosen to be a reversed pyramid. For practical reasons concerning mainly

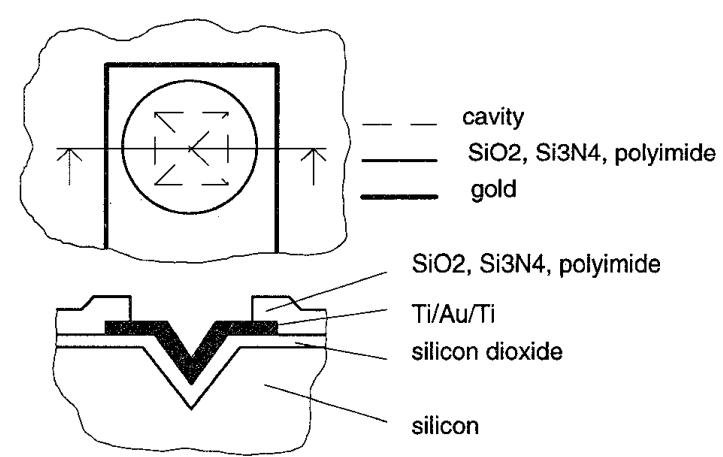

Fig. 1. Schematic representation of a MicroCavity Electrode (MCE) having one bubble nucleation site. Top and cross-sectional view

the reproducibility of the fabrication process, the MCEs were made in silicon technology. A top and a crosssectional view of a MCE are schematically presented in fig. 1 .

Silicon wafers with a $<100>$ crystal orientation were used. The microcavities were etched in $\mathrm{KOH}$ ( $33 \%$ wt.) through a $\mathrm{SiO}_{2}$ mask. The advantage is that pyramid shaped cavities with very reproducible characteristics can be anisotropically etched in silicon. The shape of the structure is fixed to a reversed pyramid with a square basis. Only the aperture of the cavity can be independently changed, resulting in a certain cavity depth.

It was mentioned in literature $[9,10]$ that gas bubbles grown by electrolysis on microelectrodes have diameters in the order of 10-200 $\mu \mathrm{m}$. To generate bubbles in a cavity, the aperture of the cavity should be comparable in size with the bubbles. The aperture was chosen to be a square with an edge of $40 \mu \mathrm{m}$.

A sandwich of $\mathrm{Ti} / \mathrm{Au} / \mathrm{Ti}$ with a thickness of $25 / 250 / 25$ $\mathrm{nm}$, respectively, was evaporated and patterned on the wafers. The active area of the MCEs was defined with a sandwich of $\mathrm{SiO}_{2}, \mathrm{Si}_{3} \mathrm{~N}_{4}$, and photo curable polyimide. At the end of this patterning process, the titanium top layer was also removed, allowing the gold surface a free contact with the aqueous solution. To detect the bubbles evolving at the microelectrode by means of overpotential or impedance fluctuations, the size of the electrode active area has to be also comparable with the bubble size. The active area of the microelectrodes used during the experiments was determined by the $\mathrm{SiO}_{2}, \mathrm{Si}_{3} \mathrm{~N}_{4}$ polyimide window, a square of $60 \times 60 \mu \mathrm{m}^{2}$. It should be noted that the polyimide cannot be directly deposited on the electrode metal because of its capability to undergo redox reactions in electrolyte solutions [11]. The $\mathrm{SiO}_{2}, \mathrm{Si}_{3} \mathrm{~N}_{4}$ layer was included to avoid this problem. The wafers were then cut into pieces and the chips were mounted on $8 \times 100 \mathrm{~mm}^{2}$ printed circuit board carriers and then encapsulated in epoxy resin. 


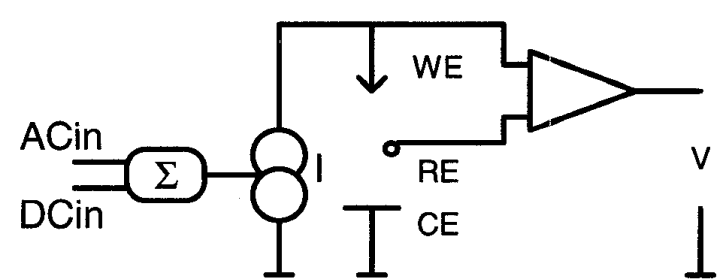

Fig. 2. Measurement set-up. WE is the working electrode, $C E$ is the counter electrode, $R E$ is the reference electrode. $I$ is the electrolysis current of a voltage controlled current source. ACin and DCin are the control voltages applied to the current source by means of the summing amplifier $\Sigma . V$ corresponds to the measured overpotential and impedance fluctuations.

\section{Measurement Set-Up}

The MCEs were used as cathodes and hydrogen bubbles were produced, according to the hydrogen evolution reaction (in alkaline medium):

cathode: $\quad 4 \mathrm{e}^{-}+4 \mathrm{H}_{2} \mathrm{O} \rightarrow 2 \mathrm{H}_{2}+4 \mathrm{OH}^{-}$

The MCEs were used as working electrodes with the set-up schematically presented in fig. 2. A platinum plate with a much bigger area than the working electrode (approx. $1 \mathrm{~cm}^{2}$ ) was used as counter electrode. A current source was connected between the working electrode and the counter electrode. The overpotential fluctuations were measured between the working electrode and a calomel reference electrode. The instrumentation amplifier was specially designed for this application and it automatically removed the $\mathrm{DC}$ component of the electrode overpotential allowing only the overpotential fluctuations to be monitored. The instrumentation amplifier also allowed the measurement of the electrode impedance variations due to the bubble evolution process. For this purpose, a signal with a frequency of $100 \mathrm{kHz}$ and a small amplitude (typically $50 \mathrm{mVpp}$ ) was added to the control voltage of the current source by means of a summing amplifier, $\Sigma$. The amplitude of this small signal voltage was chosen in such a way that also the fluctuations of the resulting current could be considered small signal. The measurements were done in $10 \mathrm{mM}$ sodium acetate $(\mathrm{NaAc})$ or sodium sulphate $\left(\mathrm{NaSO}_{4}\right)$ solutions with different nonionic surfactant concentrations (synperonic NP9).

\section{RESULTS AND DISCUSSION}

As a consequence of the electrode shaping and of the superposition of the concentration profiles caused by diffusion from the walls of the cavity, a higher dissolved gas supersaturation is obtained on the bottom of the cavity than in the bulk of the aqueous solution. Due to this higher concentration of dissolved gas, heterogeneous nucleation will take place on the bottom of the cavity resulting in one single bubble nucleation site. The cavity acts like a gas concentrator. The presence of the gas bubbles on the electrode induces changes both in the electrode overpotential and in the impedance. By monitoring one of

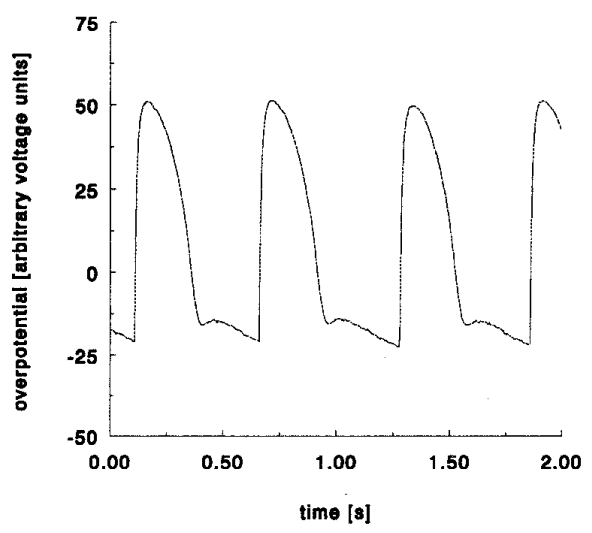

Fig. 3. Typical wave form of the measured overpotential fluctuations on MCEs. The frequency is $0.56 \mathrm{~Hz}$. The surfactant concentration was $0.8 \mathrm{~g} / \mathrm{l}$. The supporting electrolyte was NaAc, $10 \mathrm{mM}$. The electrolysis current was $5.93 \mu A$.

these changes, the evolution of bubbles can be detected with the same electrode used for bubble generation. A typical measured wave form of the overpotential is presented in fig. 3 and of the impedance fluctuations in fig. 4. The corresponding frequency of the bubbles can be easily determined from the wave forms. The mechanisms explaining the exact shape of the wave form are beyond the scope of this paper and will be discussed elsewhere.

The gas bubbles generated by electrolysis at MCEs can be used for the measurement of the dynamic surface tension in the aqueous solution if a constant electrolysis current is used. The constant electrolysis current

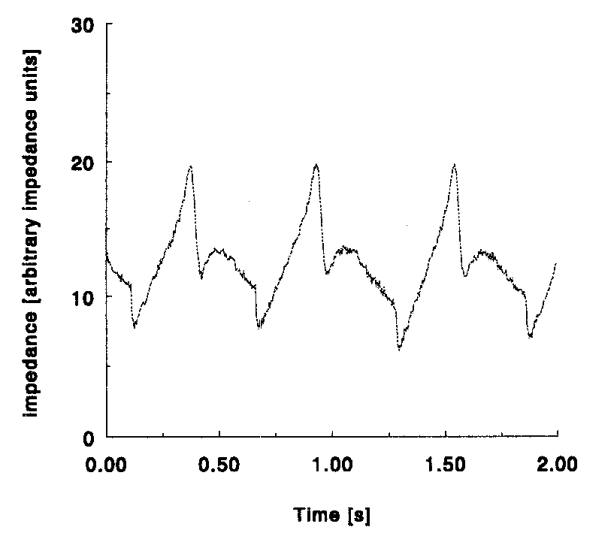

Fig. 4. Typical wave form of the impedance fluctuations on a MCE. Same process and conditions as for fig. 3. 


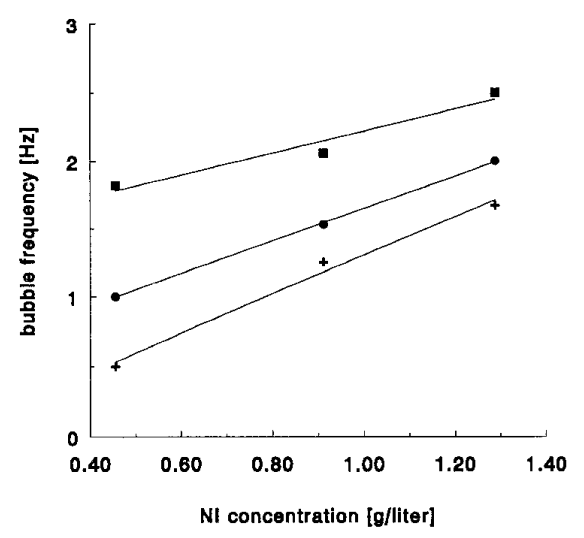

Fig. 5. Measured dependency of the bubble frequency on the surfactant concentration (dynamic surface tension) at various electrolysis currents. Measurements in $10 \mathrm{mM}$ $\mathrm{Na}_{2} \mathrm{SO}_{4}$ and with various concentrations of nonionic surfactant (synperonic NP9). The electrolysis current is:

$6 \mu \mathrm{A} ; \bullet 5.3 \mu \mathrm{A} ;+4.8 \mu \mathrm{A}$.

corresponds to a constant gas production (in analogy with the previously mentioned sparging method). Both the size and the frequency of the electrogenerated bubbles are dependent on the dynamic surface tension.

Measurement results presented in fig. 5 show a monotonous dependence of the bubble frequency on the surfactant concentration (dynamic surface tension) in the liquid under investigation. As expected from sparging, the bubble frequency increases with the increase of the surfactant concentration (and the decrease of the dynamic surface tension). Also, the bubble frequency increases if more gas is generated by a higher electrolysis current.

\section{CONCLUSION}

We conclude that the electrolytic method for in-situ generation of gas bubbles by means of MCEs is suitable for the measurement of dynamic surface tension of aqueous solutions. By means of electrode surface shaping, one single nucleation site for gas bubbles is created. The gas bubbles evolving at MCEs behave similarly to the bubbles evolving during sparging. The resulting electrolytic method is analogue to sparging, but the system is much smaller. The use of big gas pumps needed for sparging is avoided.
From the perspective of sensor development, MCEs work as actuators because they can generate ("actuate") bubbles with a controlled size on a specified place. On the other hand, also detection of the electrogenerated bubbles is possible with MCEs and thus also the sensor part is present in the same device. The overpotential and impedance measured on the MCE are related to the presence and the dimensions of the gas bubbles.

\section{ACKNOWLEDGEMENTS}

The research is financially supported by Unilever.

The authors wish to thank ir. Dion Oudejans who processed the MCE devices used for the experiments presented in this paper.

\section{REFERENCES}

[1] Van Oss, C.J, Interfacial forces in aqueous media, ed. Marcel Dekker, Inc. (1994) 14.

[2] Lubetkin, S.D. Controlled particle, droplet and bubble formation, ed. D.J. Wedlock, Butherworth \& Heinemann, ISBN 075061494 3, (1994) 159-190.

[3] Mysels, K.J. Colloids and surfaces, 43 (1990) 241-262. [4] Mueller Kirschbaum, T; Smulders, E; Hoefer, W. Patent WO 92/18680 (1992).

[5] Oh, S.G; Klein, S.P; Shah, D.O, AIChE Journal, 38-1 (1986) 149-152.

[6] Miller, T.E, Jr; Meyer, W.C, American Laboratory (1984).

[7] Westerheide, D.E; Westwater, J.W, AIChE Journal, 7 3 (1961) 357-362.

[8] Gabrielli, C; Huet, F; Keddam, M, J. Electrochem. Soc, 138-2 (1991) L82-L84.

[9] Sillen, C.W.M.P, PhD. Thesis, University of Eindhoven, (1983).

[10] Gabrielli, C; Huet, F; Keddam, M; Macias, A; Sahar, A, J. Appl. Electrochem. 19 (1989) 617-629.

[11] Lian, S.M; Chen, K.M; Hung, A. J. Electrochem. Soc. 141-9 (1994) 2374-2379. 